\title{
L-type Voltage-gated Calcium Channel Modulators Inhibit Glutamate-induced Morphology in Astrocytoma Cells
}

\author{
Mitra Sadat Tabatabaee ${ }^{1,2} \cdot$ Frederic Menard $^{1,3 *}$ \\ ${ }^{1}$ Department of Biochemistry \& Molecular Biology, I.K. Barber School of Arts \& Sciences, University of British Colombia, Kelowna, BC \\ Canada \\ ${ }^{2}$ Department of Biology, Faculty of science, Islamic Azad university, central Tehran branch, Tehran, Iran \\ ${ }^{3}$ Department of Chemistry, I.K. Barber School of Arts \& Sciences, University of British Colombia, Kelowna, BC Canada
}

\begin{abstract}
The excitatory neurotransmitter glutamate evokes physiological responses within the astrocytic network that lead to fine morphological dynamics. However, the mechanism by which astrocytes couple glutamate sensing with cellular calcium rise remains unclear. Employing natural properties of U118-MG astrocytoma cells, we tested a possible connection between L-type voltage-gated calcium channels $\left(\mathrm{Ca}_{\mathrm{v}}\right)$ and glutamate receptors. Using live confocal imaging and pharmacological inhibitors, the extension of U118-MG processes upon glutamate exposure are shown to depend mainly on extracellular calcium entry via L-type Cav's. Inhibitors of the $\mathrm{Ca}_{\mathrm{v}} \alpha 1$ protein, decreased astrocytic filopodia extension; while, gabapentinoids, ligands of the $\mathrm{Cav}_{\mathrm{v}} \mathrm{s} \alpha 2 \delta$ auxiliary subunit blocked all process growth. This study suggests that $\alpha 2 \delta$ is the main contributor to Cav's role in glutamate-dependent filopodiagenesis. It opens new avenues of research on the role of $\alpha 2 \delta$ in neuron-astrocyte glutamate signaling and neurochemical signaling at tripartite synapses.
\end{abstract}

Keywords Astrocytes; glutamate; voltage-gated calcium channels; filopodiagenesis

\section{INTRODUCTION}

Astrocytes have historically been described as nonexcitable cells that do not contribute actively to information processing in the brain, in contrast to neurons who communicate actively through electrical or chemical synapses. However, this simplistic view has evolved over the past two decades as more detailed studies on astrocytes have emerged (Araque et al. 1999; Cornell-Bell, Thomas, and Caffrey 1992; Rose et al. 2018; Bazargani and Attwell 2016). For instance, astrocytes are now known to respond to glutamate among other stimuli (Cornell-Bell, Prem, and Smith 1990; Rose et al. 2018; Cornell-Bell, Thomas, and Caffrey 1992). This astrocytic glutamate sensitivity led to the tripartite synapse model, where astrocytes are active participants in a functional neural connection (Araque et al. 1999). However, the molecular mechanisms underlying this signaling are still not fully understood (Rose et al. 2018).
Glutamate is the main excitatory neurotransmitter released at neuronal synapses (Rose et al. 2018). In astrocytes, glutamate triggers an intracellular calcium rise, followed by a rearrangement of the actin cytoskeleton, which results in movement, formation, and protrusion of filopodia (Cornell-Bell, Thomas, and Caffrey 1992). These filopodia in close proximity to synapses in vivo are termed peripheral astrocytic processes (PAPs) (Molotkov et al. 2013; Santello, Toni, and Volterra 2019). They are highly motile structures that seek to contact synapses (Haber, Zhou, and Murai 2006). Several factors have been demonstrated to influence PAPs motility and filopodia formation: neuronal activity, synapse-specificity, mGluR5 presence, $\mathrm{Ca}^{2+}$ concentration, and $\mathrm{IP}_{3}$ concentration (Bernardinelli et al. 2014; Perez-Alvarez et al. 2014; Heller and Rusakov 2015).

Filopodia formation in astrocytes upon glutamate stimulation has been proposed to occur via two main pathways based on both ionotropic glutamate receptors 


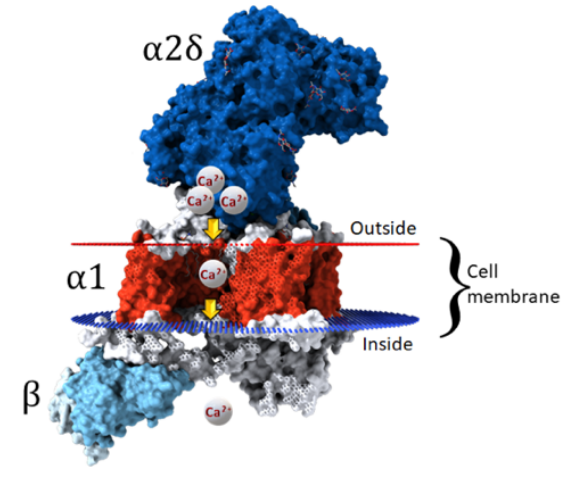

Figure 1. Assembly of L-type Cav channels. The a1 subunit (red) is composed of 24 transmembrane helices that form the selective $\mathrm{Ca}^{2+}$ pore. The intracellular $B$ subunit (light blue) is responsible for the channels trafficking to the membrane. The extracellular $a 2 \delta$ subunit (dark blue) is the modulator of a1 subunit conductance and controls the localization of the channel on the cell membrane (modified from PDB structure 5GJV).

(iGluR) and metabotropic glutamate receptors (mGluR) (Cornell-Bell, Thomas, and Caffrey 1992; Rose et al. 2018; Heller and Rusakov 2015; Matyash and Kettenmann 2010). The most abundant astrocytic iGluRs are the calcium-permeable AMPA receptors, and they have been proposed to control glutamate-dependent filopodiagenesis (Bernardinelli, Muller, and Nikonenko 2014; Heller and Rusakov 2015). Alternatively, mGluR5 has also been proposed to be the main receptor responsible for this phenomenon (Bernardinelli et al. 2014; Heller and Rusakov 2015; Panatier and Robitaille 2016). In both cases, glutamate sensing was shown to correlate with an intracellular calcium rise in astrocytes. With $\mathrm{Ca}^{2+}$-permeable AMPA receptors, glutamate causes a calcium influx from the extracellular milieu (lino et al. 2001). With mGluR5, exposure to glutamate releases $\mathrm{Ca}^{2+}$ from internal stores through 1,4,5-inositol trisphosphate $\left(\mathrm{IP}_{3}\right)$ signaling (Panatier and Robitaille 2016). In both pathways, the calcium rise leads to actin cytoskeletal rearrangement combined to filopodiagenesis, processes extension and motility (Heller and Rusakov 2015).

During the course of an unrelated study, we observed that glutamate-induced filopodiagenesis was affected by voltage-gated $\mathrm{Ca}^{2+}$ channel (Cav) modulators in the astrocytoma cell line U118-MG. The human protein atlas shows that U118-MG express only two types of glutamate receptors: mGluR8 and an isoform of AMPA receptor possessing iGluR2 subunits (GRIA2). Neither of these can directly link glutamate sensing with the calcium rise that mediates the filopodia extension. Consequently, we investigated whether a connection exists between glutamate sensing and high voltage-gated calcium channels (L-type Cav's) in U118-MG cells.

Cav's are classified according to their electrophysiological and pharmacological properties into high Cav's (L-, N-, P-, Q-, and R-type) and low Cav's (Ttype) (Latour et al. 2003; Zamponi et al. 2015). Each channel couples a change in membrane potential to a $\mathrm{Ca}^{2+}$ influx, where $\mathrm{Ca}^{2+}$ ions act as secondary messengers in events such as contraction, secretion, and gene expression (Woolley et al. 1990). Structurally, they consist of three main subunits that associate in a $1: 1: 1$ stoichiometry: a pore-forming $\alpha 1$ subunit, an external $\alpha 2 \delta$ subunit, and an intercellular $\beta$ subunit (Figure1) (Zamponi et al. 2015; Thul et al. 2017). The $\beta$ subunit ensures the $\mathrm{Ca} v$ channel localizes to the membrane, while the $\alpha 1$ and $\alpha 2 \delta$ subunits regulate ion conductance. The transmembrane $\alpha 1$ forms the calcium channel pore and contains the voltage sensor. The extracellular $\alpha 2 \delta$ subunit modulates properties of $\alpha 1$ such as its activation and conductance rate (Woolley et al. 1990; Striessnig and Koschak 2008; Song et al. 2015).

Astrocytes are not electrically excitable cells, yet they express L-type $\mathrm{Ca}_{\mathrm{v}}$ 's (Latour et al. 2003). The exact function of these voltage-dependent channels in astrocytes is still unknown, but their contribution in pathological conditions of the brain has been extensively demonstrated, with Cav1.2 in particular (Cheli et al. 2016; Wang et al. 2015; Westenbroek et al. 1998; Anekonda and Quinn 2011; Shaw and Colecraft 2013; Daschil et al. 2013). The only L-type Cav shown to be expressed in U118-MG cells is Cav1.2 (Thul et al. 2017).

We originally hypothesized that Cav might participate to filopodiagenesis in astrocytes when the cells are subjected to glutamate stimulation by amplifying intracellular $\mathrm{Ca}^{2+}$ concentrations after initial activation of iGuR and/or mGluR. iGluRs and phospholipase Clinked mGluRs (group II and III) are known to cause an intracellular $\mathrm{Ca}^{2+}$ rise upon glutamate binding that can lead to the gating of Cav's (Verkhratsky and Kirchhoff 2007). Adenylate cyclase-linked mGluRs (group I) can also trigger the gating of Cav's by activating the channel through phosphorylation (Hove-Madsen et al. 1996). In both cases, gating of Cavs can provide enough calcium to support the cytoskeletal reorganization that leads to a morphological change. Therefore, we expected that 
interrupting calcium conductance of $\mathrm{Ca}$ v would inhibit or decrease the morphological response to glutamate in astrocytes.

In this study, we initially showed that the calcium required for filopodiagenesis in response to glutamate is supplied from the extracellular milieu, which suggests an active role of membrane ion channels. Next, to test our hypothesis on the role of Cav's, we employed antagonists for the pore-forming $\alpha 1$ subunit of L-type calcium channels and ligands selective for its $\alpha 2 \delta$ subunit. We were surprised to find that the effect of $\alpha 2 \delta$ ligands was stronger than the effect of $\alpha 1$ antagonists. In fact, gabapentinoids targeting $\alpha 2 \delta$ completely inhibited the morphological response of U118-MG cells to glutamate, while $\alpha 1$ antagonists only partially decreased the cellular response. Herein, we present evidence suggesting that the $\alpha 2 \delta$ auxiliary subunit is the main contributor to $\mathrm{Cav}$ role in the glutamate-dependent filopodiagenesis of astrocytes.

\section{MATERIALS AND METHODS}

\section{Cell culture}

Human astrocytoma U118-MG cell line (HTB-15, ATCC) was gifted by Dr. Andis Klegeris, UBC Okanagan. U118-MG cells were cultured in Dulbecco's modified essential medium (DMEM, Gibco 11995-065) supplemented with $10 \% \mathrm{v} / \mathrm{v}$ heat-inactivated fetal bovine serum (HyClone 12483020) 1\% v/v penicillin 10,000 $\mathrm{U} / \mathrm{ml}$ and streptomycin 10,000 $\mu \mathrm{g} / \mathrm{ml}$ (Gibco 15140163). Cells were incubated in a humidified atmosphere containing $5 \% \mathrm{CO}_{2}$ at $37^{\circ} \mathrm{C}$, and typically passaged at 80 $95 \%$ confluence using a pre-warmed $0.25 \%$ trypsinEDTA solution for a maximum of 5 minutes (Gibco SH30236.02).

For experiments, cells from a $10 \mathrm{~cm}$ culture dish at $\sim 80 \%$ confluence were resuspended with a pre-warmed $0.25 \%$ trypsin-EDTA solution and transferred to $35 \mathrm{~mm}$ glass-bottom culture dishes (Mutsunami $\mathrm{D} 1130 \mathrm{H}$ ) in phenol red-free, high glucose DMEM supplemented with 25 mM HEPES (Gibco 21063-029). Enough cells were transferred to obtain a $\sim 50 \%$ confluent culture dish after adhesion. Cells were incubated for less than the cells' doubling time, typically $\sim 33 \mathrm{~h}$ to minimize imaging variation between samples (see statistical analysis section) (Westermark 1973). This period allows cells to adopt a flattened shape that helps define the filopodia for measurements. Identical incubation times were respected to ensure cells were at the same growing stage in all treatments.

Prior to imaging, the medium was saturated with $\mathrm{CO}_{2}$ bubbling delivered via a needle attached to a $0.22 \mu \mathrm{m}$ syringe filter. $\mathrm{CO}_{2}$ maintain U118-MG viability on the microscope (Aumann et al. 2017).

\section{Transfection}

U118-MG astrocytoma cells were transfected with 1200 $\mathrm{ng} / \mu \mathrm{l}$ of the F-actin marker mCherry-LifeAct-7 plasmid using a calcium phosphate precipitation protocol (Kingston, Chen, and Rose 2003). mCherry-Lifeact-7 was a gift from Michael Davidson (Addgene plasmid \# 54491)

\section{Cells treatment}

On the microscope stage, U118-MG cells in phenol redfree, high glucose DMEM supplemented with $25 \mathrm{mM}$ HEPES (Gibco 21063-029) were stimulated with glutamate (Alfa Aesar A15031-30). Briefly, $10.0 \mu \mathrm{L}$ of a 20X glutamate stock solutions as carefully delivered to the medium ( $2.0 \mathrm{~mL}$ in a $35 \mathrm{~mm}$ dish) at the outside edge of the dish for a final concentration of $100 \mu \mathrm{M}$. The stock solution of L-glutamate was prepared in deionized millipore water and filter-sterilized through a $0.22 \mu \mathrm{m}$ syringe filter (Cornell-Bell, Prem, and Smith 1990; Aumann et al. 2017).

For $\mathrm{Ca}^{2+}$-blocking experiments, $1.00 \mu \mathrm{L}$ of a $200 \mathrm{X}$ stock solution of $\mathrm{Ca}_{\mathrm{v}}$ inhibitor was delivered to the glassbottom culture dishes (Mutsunami D11130H) for a final concentration of each reagent as shown in Table 1. After each addition, cells were incubated at $37{ }^{\circ} \mathrm{C}$ under a humidified $5 \% \mathrm{CO}_{2}$ atmosphere for the time indicated in Table 1 ( 4 minutes to 3 hours).

Stock solutions of $\mathrm{Ca}_{v}$ inhibitors were prepared in DMSO (vehicle) before each experiment. Solutions were filtersterilized using a $0.22 \mu \mathrm{m}$ syringe filter. To avoid negative effect of DMSO on the cells, stock solutions were highly concentrated (200X), thereby maintaining the final amount of DMSO below $1 \% \mathrm{v} / \mathrm{v}$ in the media. The exception were gabapentinoids, which were prepared directly in deionized millipore water vehicle, also as a 200X stock solution for consistency. 

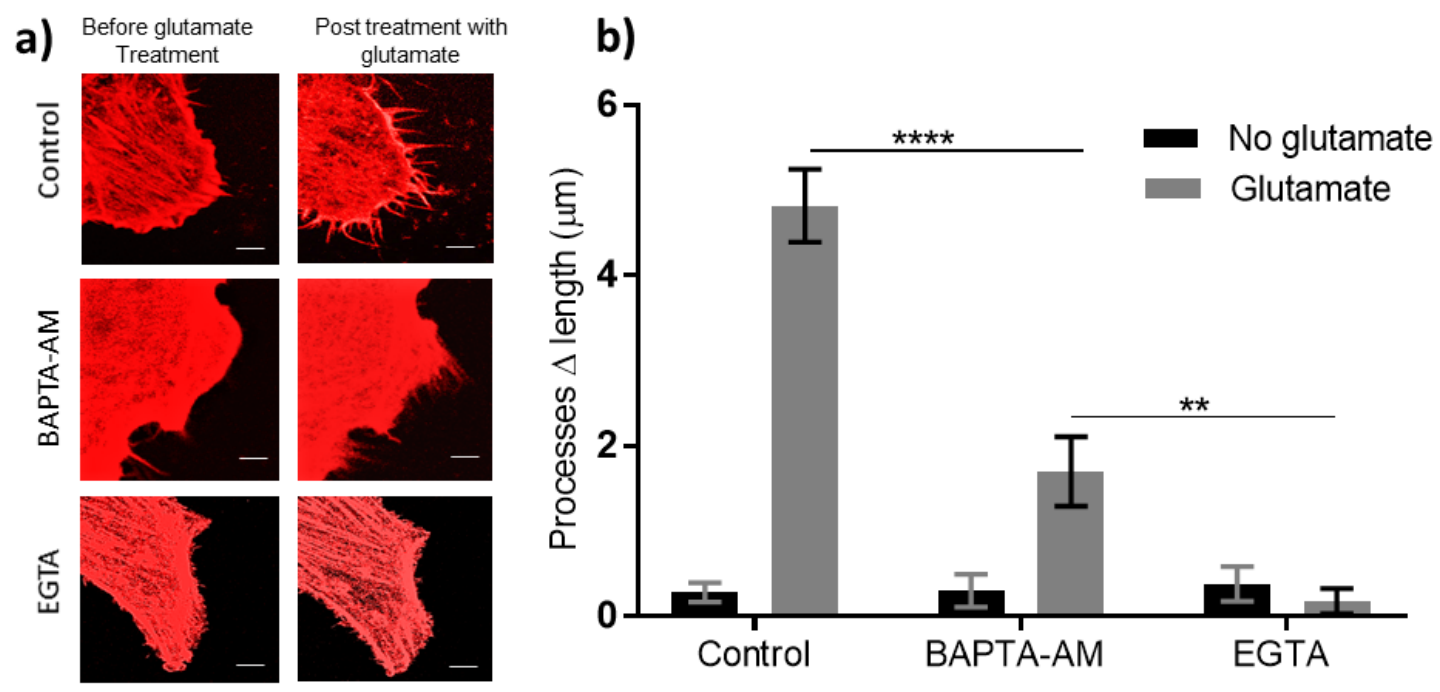

Figure 2. Extracellular calcium is essential for glutamate-induced filopodia extension in model astrocytes. (a) Representative images of U118-MG astrocytoma cells upon exposure to $100 \mu \mathrm{M}$ glutamate for 20 minutes when treated with and without intra- or extracellular calcium chelators. Confocal fluorescence micrographs of cells transfected with mCherry-LifeAct7 at 60X magnification; scale bar is $5 \mu \mathrm{m}$. (b) Cells extend their processes significantly in response to $100 \mu \mathrm{M}$ glutamate. Chelating intracellular $\mathrm{Ca}^{2+}\left(10 \mu \mathrm{M}\right.$ BAPTA-AM) or extracellular Ca ${ }^{2+}$ (50 $\mu \mathrm{M}$ EGTA) decreases the length of processes extension induced by $100 \mu \mathrm{M}$ glutamate. Chelating the intracellular calcium with BAPTAAM still allowed for significant process extension, yet it is significantly shorter than that of the control cells with glutamate. In contrast, chelating the extracellular calcium with EGTA inhibited filopodia growth. Change in length $(\Delta)$ was calculated by subtracting the length of a filopodium immediately prior to stimulation from that of the same filopodium after 20 minutes. Results are presented as mean \pm SEM. The change in length is the average data of 5 cells, each from a separate culture dish; measurements of at least 20 filopodia were averaged for each cell ( $\mathrm{n}$ $=5$ ). All comparisons use a post hoc Tukey's multiple comparison (two-way ANOVA, $\mathrm{F}_{2,24}$ glutamate treatment $=34.98, \mathrm{~F}_{2,24} \mathrm{Ca}^{2+}$ chelators $=71.38$, for both factors $p<0.0001),{ }^{* *} p=0.002,{ }^{* * *} p<0.0001$.

For calcium chelation experiments, either intracellular or extracellular, the same protocol as for Cav inhibitors was used with times and concentrations listed in Table 1. Confocal microscopy and live imaging

Imaging of live cells was conducted on an Olympus FV1000 fluorescence confocal microscope equipped with a Plan-ApoN 60x/i.4 oil-immersion objective. Fluorescence of mCherry-Lifeact-7 was observed at 559 $\mathrm{nm}$ excitation using a He-Ne laser (Olympus filter set Су3.5).

Changes in cell morphology were recorded at $60 \mathrm{X}$ magnification. To measure filopodia extension, an image of a single cell was acquired every 20 second for a total of number of 60 frames.

\section{Quantification of filopodia extension}

The length of at least twenty filopodia per cell was measured manually using FIJI image analysis software. Only filopodia whose arbor could be unambiguously visualized throughout the entire imaging period were selected (Zatkova et al. 2018). A filopodium's length was measured from the edge of the cell membrane to its apical end; each manual measurement was repeated three times on the same image. The change in length was calculated by subtracting the length of a process prior to stimulation (frame 10; three minutes) from the length of the same filopodium after 20 minutes (frame 60). Glutamate was delivered to the medium four minutes after the beginning of imaging (i.e., frame 12). The reported "change in length" for one cell represents the average of 20 measured filopodia per cell.

\section{Statistical analysis}

Each value represents the averaged changes measured from at least 5 cells, each from a separate culture dish (n $\geq 5$ ); the results are presented as the mean \pm S.E.M. Significant differences among groups were determined using one-way or two-way analysis of variance (ANOVA) and post hoc analysis using Prism 7.04 from GraphPad. A $p$ value $<0.05$ was considered significant. Statistically non-significant data is not starred on plots. To minimize technical variations within our samples for ANOVA analysis, we employed a completely randomized 


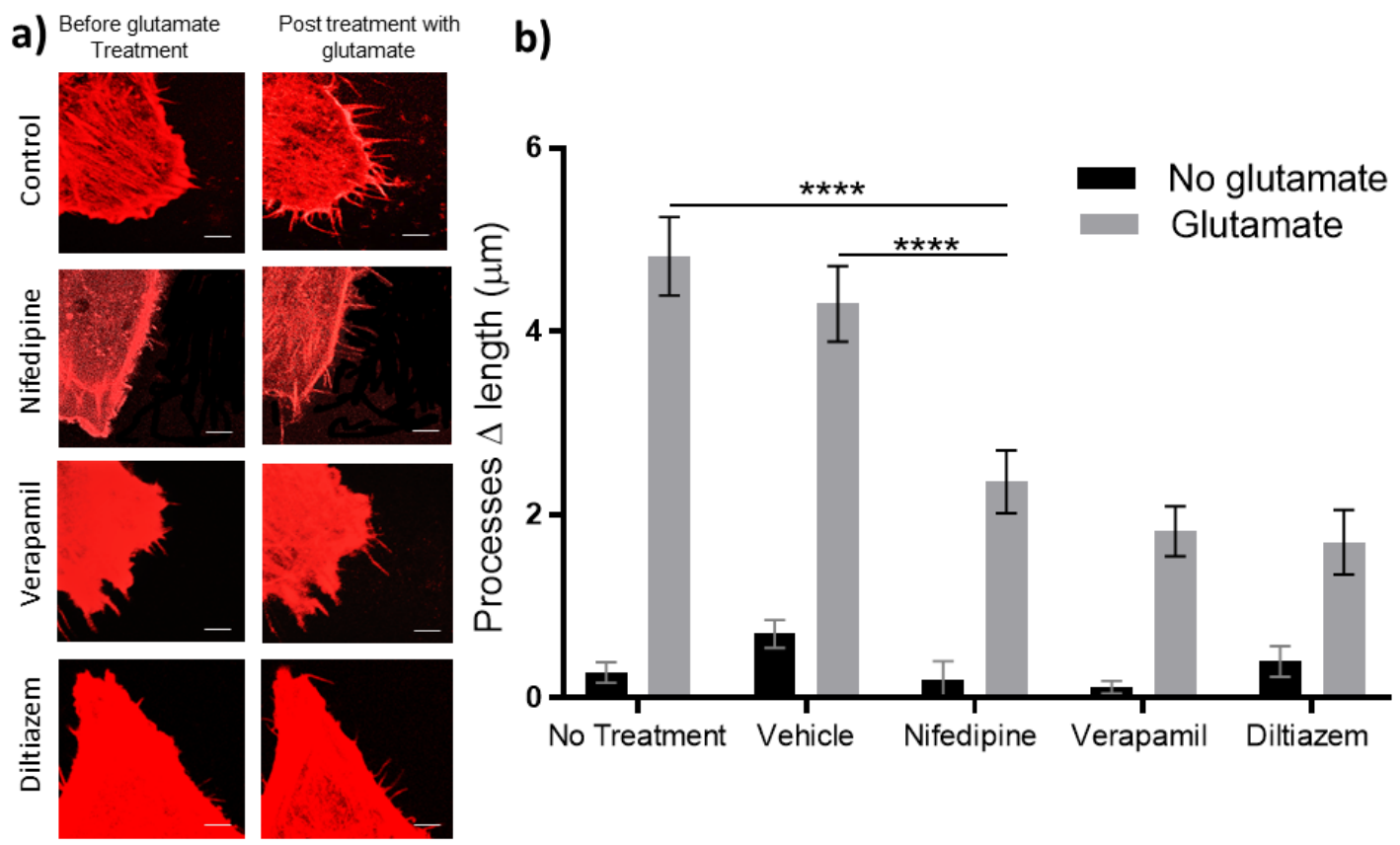

Figure 3. Blocking the pore-forming subunit of L-type Cav's significantly decreases processes extension induced by glutamate in U118 cells. (a) Representative images of U118-MG astrocytoma cells upon exposure to $100 \mu \mathrm{M}$ glutamate after being treated with L-type Cav blockers $(10 \mu \mathrm{M})$ for 30 minutes prior to stimulation. Confocal fluorescence micrographs of cells transfected with mCherry-LifeAct7 at $60 \mathrm{X}$ magnification; scale bar is $5 \mu \mathrm{m}$. (b) Blocking the pore-forming subunit of L-type calcium channels significantly decreases the processes extension upon exposure to glutamate. All treatments with glutamate caused a significant increase in processes length compared to unstimulated cells. Processes extension was significantly decreased when cells were treated with L-type Cav blockers prior to glutamate exposure. Vehicle solution was DMSO only. Change in length $(\Delta)$ was calculated by subtracting the length of a filopodium immediately prior to stimulation from that of the same filopodium after 20 minutes. Results are presented as mean \pm SEM. The change in length is the average data of 5 cells, each from a separate culture dish; measurements of at least 20 filopodia were averaged for each cell $(n=5)$. Data were analyzed by two-way ANOVA $\left(F_{4,40}\right.$ glutamate treatment $=16, F_{1,40} \mathrm{Ca}^{2+}$ blockers $=227.3$, for both factors $\left.p<0.0001\right)$. All comparisons done by post hoc Tukey's multiple comparison.

block design (Krzywinski and Altman 2014). Briefly: cells of a dish at $80-95 \%$ confluency were trypsinized and simultaneously re-plated in several $35 \mathrm{~mm}$ imaging dishes and incubated in a humidified atmosphere containing 5\% $\mathrm{CO}_{2}$ at $37^{\circ} \mathrm{C}$ for less than 33 hours to avoid variation between samples.

\section{RESULTS}

\section{U118-MG cells extend their processes in exposure to glutamate}

When astrocytoma cells were imaged in their medium without stimulation, their filopodia length changed by $0.28 \pm 0.24 \mu \mathrm{m}$ over the course of 16 minutes. In contrast, when cells were subjected to $100 \mu \mathrm{M}$ glutamate (4 min. after beginning of imaging), their processes grew by 4.82 $\pm 0.95 \mu \mathrm{M}$ (Fig. 1B). This 17 -fold increase in length was statistically significant $(\mathrm{p}>0.0001)$. A rapid process extension was observable within the first two to five minutes after glutamate exposure (not shown), but the final length filopodia were measured 16 minutes poststimulation to allow cells to stabilize.

\section{The glutamate-induced process extension in U118- MG cells depends on extracellular calcium}

Chelation of extracellular free $\mathrm{Ca}^{2+}$ ions with EGTA in the medium completely abolished filopodia extension upon exposure to $100 \mu \mathrm{M}$ glutamate $(p<0.0001$, Fig. $1 B)$. In presence of EGTA, the change in length is statistically indistinguishable from the baseline cell response. However, when the intracellular free $\mathrm{Ca}^{2+}$ was 

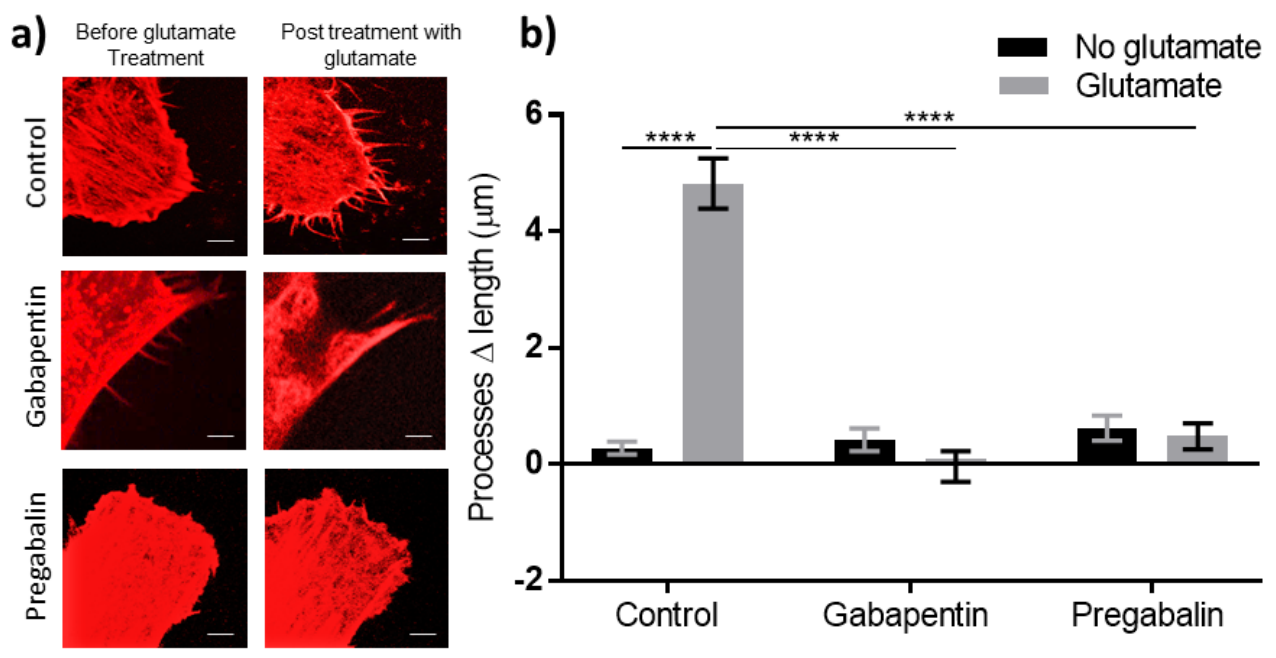

Figure 4. The $a_{2 \delta}$ subunit of L-type Cav controls filopodia extension when astrocytoma cells are exposed to glutamate. (a) Representative images of U118-MG cells exposed to $100 \mu \mathrm{M}$ glutamate after being treated (or not) with $10 \mu \mathrm{M}$ a2 $\delta$ subunit inhibitors for 30 minutes prior to glutamate stimulation. Confocal fluorescence micrographs of cells transfected with mCherry-LifeAct7 at 60X magnification; scale bar is $5 \mu \mathrm{m}$. (b) Pharmacologically blocking the a2ठ subunit of L-type Cav's with gabapentinoids completely inhibited filopodia growth or extension in response to $100 \mu \mathrm{M}$ glutamate. Change in length $(\Delta)$ was calculated by subtracting the length of a filopodium immediately prior to stimulation from that of the same filopodium after 20 minutes. Results are presented as mean \pm SEM. The change in length is the average data of 5 cells, each from a separate culture dish; Measurements of at least 20 filopodia were averaged for each cell $(n=5)$. Data were analyzed by two-way ANOVA $\left(F_{1,24}\right.$ glutamate treatment $=38.75, F_{2,24}$ treatments $=48.72$, for both factors $\left.p<0.0001\right)$; data comparisons used a post hoc Dunnet multiple comparison $(p<0.0001)$.

sequestered with BAPTA-AM, the cell's response to glutamate stimulation was significantly reduced, but not completely inhibited: filopodia extension was about $35 \%$ of that observed with control cells $(p<0.001$, two-way ANOVA post hoc Sidak's multiple comparison tests).

\section{Blocking the pore-forming subunit of L-type Cav's does not block the morphological response of astrocytoma cells to glutamate}

Figure 2 displays the effect of subjecting U118-MG cells to drugs known to inhibit $\mathrm{Ca}^{2+}$ ion influx by interacting with the $\alpha 1$ subunit of L-type $\mathrm{Ca}_{\mathrm{v}}$ 's. Upon glutamate stimulation, the processes extension decreased significantly $(p<0.0001)$, but was still observed to be 30$50 \%$ of the negative control with unblocked cells. More specifically, when cells were pre-treated with drugs for 30 minutes prior to glutamate stimulation, the average increase in processes length was: $2.36 \pm 0.77 \mu \mathrm{m}$ with 10 $\mu \mathrm{M}$ nifedipine, $1.82 \pm 0.60 \mu \mathrm{m}$ with $10 \mu \mathrm{M}$ verapamil, and $1.70 \pm 0.79 \mu \mathrm{m}$ with $10 \mu \mathrm{M}$ ditiazem. $\mathrm{Ca}_{\mathrm{v}} \alpha 1-$ targeting inhibitors decreased filopodia protrusion by 50 $65 \%$ compared to the positive controls, i.e., untreated cells $(4.82 \pm 0.95 \mu \mathrm{m})$ or vehicle treatment $(4.30 \pm 0.92$ $\mu \mathrm{m})$.

\section{Antagonists of L-type Cav's a2 $\delta$ subunit block the morphological response of astrocytoma cells to glutamate}

Processes extension and filopodiagenesis were completely inhibited when U118-MG cells were pretreated with gabapentinoids drugs for 30 minutes, prior to glutamate exposure (Fig. 3). When $10 \mu \mathrm{M}$ solutions of either gabapentin or pregabalin in water were used, no filopodia extension was observed upon glutamate stimulation ( $p>0.8$, one-way ANOVA Dunnett's multiple comparison post hoc test). The changes in processes length were statistically indistinguishable from the background growth without glutamate stimulation. 


\section{DISCUSSION}

In this study, L-type voltage-gated calcium channels (Cav) are shown to play a significant role in the glutamate-induced filopodiagenesis of astrocyte model cells U118-MG. Of the three protein subtypes constituting L-type $\mathrm{Cav}$, the $\alpha 2 \delta$ subunit appears essential to control filopodia extension, while the transmembrane channel $\alpha 1$ subunit may only be partially involved. These results are consistent with what has been observed in primary astrocytes (Cornell-Bell, Thomas, and Caffrey 1992; Cornell-Bell, Prem, and Smith 1990; Araque et al. 1999; Bernardinelli et al. 2014; Aumann et al. 2017). U118-MG is a well-established human astrocyte model cells, derived from malignant gliomas by Ponten in the late 1960's along with U138-MG. The transcriptome of U118-MG and U138-MG has been characterized; they have identical variable number tandem repeat (VNTR) and similar short tandem repeat (STR) patterns (Uhlen et al. 2010).

In our study, sequestering the extracellular calcium during glutamate stimulation prevented the protrusion of cell processes; while, sequestering the intracellular calcium still elicited about a third of the response (Fig. 1). Although it suggests that both intracellular and extracellular $\mathrm{Ca}^{2+}$ contribute to processes extension, it indicates that the calcium-mediated cytoskeletal rearrangement is more likely initiated via membrane channels rather than from intracellular stores. A concentration ffect may also be taking place: a sustained influx from the medium may provide enough $\mathrm{Ca}^{2+}$ ion to elicit maximal morphological response, while a limited intracellular amount of calcium would only lead to a lesser extension. This implication makes the group I mGluRs, that induce intracellular calcium release from internal stores via the $\mathrm{IP}_{3}$ cascade, unlikely to be involved in processes extension caused by glutamate in U118-MG cells. This contrasts with the studies that have proposed mGluR5 (a group I mGluR) to be the main receptor in astrocytic glutamate signaling (Rose et al. 2018), and was found to be part of glutamate signaling in hippocampal astrocytes (Porter and McCarthy 1996; Latour et al. 2003; Panatier and Robitaille 2016). However, databases show the type of astrocytoma cell lines that we are using do not express GluR5 under normal cell culture conditions (Thul et al. 2017). In addition, our data acquired from U118-MG cells showing that extracellular calcium induces stronger filopodiagenesis, support previous studies that have questioned the function of astrocytic mGluR5 in glutamate signaling. For instance, mGluR5 agonists failed to induce calcium signaling in astrocytes in the adult brain (Sun et al. 2013). mGluR5 has also been reported to be only partially responsible for calcium signals in response to neural glutamate release in astrocytic processes from mature hippocampal mossy fibers in another research (Haustein et al. 2014). These studies combined to our observations suggest that a transmembrane calcium conductor must be involved within calcium events of astrocytic glutamate signaling (Oberheim et al. 2006; Verkhratsky and Kirchhoff 2007).

The membrane-bound ion channels iGluRs such as NMDA and AMPA receptors are obvious candidates for glutamate-induced filopodiagenesis. However, NMDA receptors have been indicated to be either unfunctional or absent in hippocampal cultured astrocytes or even in hippocampal slices (Matyash and Kettenmann 2010). In the type of astrocytoma cells that we are employing, the NMDA receptors shown to lack the essential NR1 subunit which makes them unfunctional (Swanson and Sakai 2009; Uhlen et al. 2010). On the other hand, calcium-permeable AMPA receptors have been reported to contribute to the calcium-dependent events associated with glutamate signaling: in Bergmann glial cells from cerebellar cortex (Matsui, Jahr, and Rubio 2005), in radial-like glial cells from the dentate gyrus subventricular zone (Swanson and Sakai 2009; Matyash and Kettenmann 2010; Rose et al. 2018), and in Bergmann glia appendages at Purkinje cell synapses (Swanson and Sakai 2009). However, U118-MG evidenced to express iGluR2 which restricts the $\mathrm{Ca}^{2+}$ conductance of AMPA receptors (Swanson and Sakai 2009; Uhlen et al. 2010) and therefore makes them unlikely to directly mediate the calcium influx required 
for cytoskeletal rearrangement following glutamate sensing.

Serendipitous observations in another part of our research program on astrocytic Cav's led us to hypothesize that they have an active role in astrocytic glutamate signaling. Despite being non-electrical cells, astrocytes express voltage-gated calcium channels, however their function in these cells is still unclear (Latour et al. 2003; Cheli et al. 2016). We initially posited that astrocytic Cav's might amplify the intracellular calcium amounts necessary for filopodiagenesis via actin reorganization. Upon glutamate stimulation, membranebound GluRs could activate Cav's by changing the membrane potential, therbery activating the voltage sensors of the pore-forming Cav's $\alpha 1$ subunit (Hille 2001; Youn, Gerber, and Sather 2013). Another possibility could involve adenylate cyclase-coupled mGluRs (mGluR8 in U118-MG (Thul et al. 2017)) that can potentiate Cav gating by phosphorylation (Hove-Madsen et al. 1996; Lepski et al. 2013).

The results from experiments with two sets of $\mathrm{Cav}$ inhibitors provide strong evidence for a functional role for astrocytic Cav's in the morphological response of astrocytes to glutamate. Three inhibitors of $\mathrm{Ca}_{\mathrm{v}} \alpha 1$ significantly decreased the extent of glutamate-induced filopodia extension in U118-MG cells, but only partially (Fig. 2). In fact, a similar role for L-type Cav's in the extension of neural dendrites has been reported recently (Kamijo et al. 2018; Zatkova et al. 2018). In these studies with neurons, the stimuli and downstream pathways differ from our study with astrocytoma cells, but the contribution of L-type Cav's to the extension of processes is consistent. The limited filopodia extension observed with $\alpha 1$ inhibitors (nifedipine, verapamil, diltiazem) could be due to a parallel calcium-entry pathway sensitive to glutamate, or to secondary messenger signaling via intracellular calcium release. However, neither would appear to be sufficient to elicit a full morphological response.

Surprisingly, the morphological response of cells to glutamate was virtually abolished when gabapentinoids were ued, as inhibitors of $\alpha 2 \delta 1-$ Cav's second subunit essential to calcium influx (Fig. 3). The extracellular $\alpha 2 \delta$ subunit of $\mathrm{Ca}_{\mathrm{v}}$ is known to modulate ion conductance of the $\alpha 1$ subunit, as well as to control its localization within the cell membrane (Woolley et al. 1990; Striessnig and Koschak 2008; Song et al. 2015; Robinson et al. 2011). In the context of neurons, $\alpha 2 \delta$ has been confirmed to directly interact with the neuronal actin structures of the cell membrane (Robinson et al. 2010, 2011). It was also shown to affect the cytoskeletal rearrangement in neurons (Kurshan, Oztan, and Schwarz 2009), and to control dendritic spine morphogenesis (Christopher Risher et al. 2018). Thus, this Cav subunit is likely to act similarly in astrocytic morphology. Furthermore, $\alpha 2 \delta$ has been demonstrated to be accumulated in brain where the excitatory glutamatergic synapses are formed. This specific localization may stregnthen a possible role in intercellular glutamate signaling events (Geisler et al. 2019). These studies converge with the results presented herein to suggest a significant role of $\alpha 2 \delta$ protein in morphological responses of fine astrocytic processes.

It should be noted that gabapentinoids have been suggested to cause a $\mathrm{Ca}^{2+}$ influx in cells by enhancing sodium/glutamate co-transport of $\mathrm{Na}^{+} / \mathrm{Ca}^{2+}$ exchanger via its reverse mode (Kirischuk, Kettenmann, and Verkhratsky 1997; Rojas et al. 2007; Yoshizumi, Eisenach, and Hayashida 2012). If this side-effect of gabapentinoids occurred here, one would expect to see an increase of processes extension due to $\mathrm{Ca}^{2+}$ influx. Instead, gabapentinoids completely inhibited the morphological response of astrocytoma cells to glutamate. This unexpected strong inhibition suggests a different and currently undefined mechanism of action for gabapentinoids in abolishing glutamate-induced filopodiagenesis.

The study presented above opens a new avenue of research to decipher the contribution of astrocytic Cav subunits in functional synapses. It may also offer new insight into how gabapentinoid drugs act in the treatment of epilepsy and neuropathic pain (Taylor, Angelotti, and Fauman 2007; Robinson et al. 2011; Dolphin 2012; Chen et al. 2018). These findings are now leading us to further investigate the role of $\alpha 2 \delta$ subunit of Cavs in PAPs 
motility and glutamate-dependent events within tripartite synapse.

Acknowledgments We are grateful to Andis Klegeris for generous gifts of cell lines.

Authors contributions MT conceived and performed the experiments, analyzed the data, and co-wrote the manuscript. FM conceived the idea and co-wrote the manuscript.

Funding This study was funded by the Natural Sciences and Engineering Research Council of Canada (NSERC RGPIN-2014-04982), and a UBC Eminence Fund (grant no. 62R10870). M.T. thanks University of British Columbia for Graduate Fellowships.

\section{REFERENCES}

Anekonda, Thimmappa S, and Joseph F Quinn. 2011. "Calcium Channel Blocking as a Therapeutic Strategy for Alzheimer's Disease: The Case for Isradipine." Biochimica et Biophysica Acta 1812 (12): 1584-90. https://doi.org/10.1016/j.bbadis.2011.08.013.

Araque, Alfonso, Vladimir Parpura, Rita P. Sanzgiri, and Philip G. Haydon. 1999. "Tripartite Synapses: Glia, the Unacknowledged Partner." Trends in Neurosciences 22 (5): 208-15. https://doi.org/10.1016/S01662236(98)01349-6.

Aumann, Georg, Felix Friedländer, Matthias Thümmler, Fabian Keil, Robert Brunkhorst, Horst-Werner Korf, and Amin Derouiche. 2017. "Quantifying Filopodia in Cultured Astrocytes by an Algorithm." Neurochemical Research 42 (6): 1795-1809. https://doi.org/10.1007/s11064-017-2193-0.

Bazargani, Narges, and David Attwell. 2016. "Astrocyte Calcium Signaling: The Third Wave." Nature Neuroscience 19 (2): 182-89. https://doi.org/10.1038/nn.4201.

Bernardinelli, Yann, Dominique Muller, and Irina Nikonenko. 2014. "Astrocyte-Synapse Structural Plasticity." Neural Plasticity 2014: 232105. https://doi.org/10.1155/2014/232105.

Bernardinelli, Yann, Jerome Randall, Elia Janett, Irina Nikonenko, Stéphane König, Emma Victoria Jones, Carmen E Flores, et al. 2014. "Activity-Dependent Structural Plasticity of Perisynaptic Astrocytic Domains Promotes Excitatory Synapse Stability." Current Biology: CB 24 (15): 1679-88. https://doi.org/10.1016/j.cub.2014.06.025.

Cheli, Veronica T, Diara A Santiago González, Jessica Smith, Vilma Spreuer, Geoffrey G Murphy, and Pablo M Paez. 2016. "L-Type Voltage-Operated Calcium

\section{COMPLIANCE WITH ETHICAL STANDARDS}

Conflict of interest The authors declare that they have no conflict of interest.

Ethical approval This study did not involve protocols requiring ethical approval.

Research involving human participants and/or animals. This Article does not contain any studies with human participants or animal performed by any of the authors.

Informed consent. Informed consent was obtained from all individual participants included in the study.

Channels Contribute to Astrocyte Activation In Vitro." Glia 64 (8): 1396-1415. https://doi.org/10.1002/glia.23013.

Chen, Jinjun, Lingyong Li, Shao Rui Chen, Hong Chen, Jing Dun Xie, Rita E. Sirrieh, David M. MacLean, et al. 2018. "The A2 $\delta$-1-NMDA Receptor Complex Is Critically Involved in Neuropathic Pain Development and Gabapentin Therapeutic Actions." Cell Reports 22 (9): 2455-68. https://doi.org/10.1016/j.celrep.2018.02.021.

Christopher Risher, W, Namsoo Kim, Sehwon Koh, JiEun Choi, Petar Mitev, Erin F Spence, Louis-Jan Pilaz, et al. 2018. "Thrombospondin Receptor A2 8 -1 Promotes Synaptogenesis and Spinogenesis via Postsynaptic Rac1." https://doi.org/10.1083/jcb.201802057.

Cornell-Bell, A H, P G Thomas, and J M Caffrey. 1992. "Ca2+ and Filopodial Responses to Glutamate in Cultured Astrocytes and Neurons." Canadian Journal of Physiology and Pharmacology 70 Suppl: S206-18. http://www.ncbi.nlm.nih.gov/pubmed/1363529.

Cornell-Bell, Ann H., Thomas G Prem, and Stephen J Smith. 1990. "The Excitatory Neurotransmitter Glutamate Causes Filopodia Formation in Cultured Hippocampal Astrocytes." Glia 3 (5): 322-34. https://doi.org/10.1002/glia.440030503.

Daschil, Nina, Gerald J Obermair, Bernhard E Flucher, Nadia Stefanova, Birgit Hutter-Paier, Manfred Windisch, Christian Humpel, and Josef Marksteiner. 2013. "CaV1.2 Calcium Channel Expression in Reactive Astrocytes Is Associated with the Formation of Amyloid- $\beta$ Plaques in an Alzheimer's Disease Mouse Model." Journal of Alzheimer's Disease : JAD 37 (2): 43951. https://doi.org/10.3233/JAD-130560.

Dolphin, Annette C. 2012. Calcium Channel A2 $\delta$ Subunits in 
Epilepsy and as Targets for Antiepileptic Drugs. Jasper's Basic Mechanisms of the Epilepsies. National Center for Biotechnology Information (US).

http://www.ncbi.nlm.nih.gov/pubmed/22787633.

Geisler, Stefanie, Clemens L Schöpf, Ruslan Stanika, Marcus Kalb, Marta Campiglio, Daniele Repetto, Larissa Traxler, Markus Missler, and Gerald J Obermair. 2019. "Presynaptic A2 $\delta$-2 Calcium Channel Subunits Regulate Postsynaptic GABAA Receptor Abundance and Axonal Wiring." The Journal of Neuroscience: The Official Journal of the Society for Neuroscience 39 (14): 2581-2605. https://doi.org/10.1523/JNEUROSCI.2234-18.2019.

Haber, Michael, Lei Zhou, and Keith K. Murai. 2006. "Cooperative Astrocyte and Dendritic Spine Dynamics at Hippocampal Excitatory Synapses." Journal of Neuroscience 26 (35): 8881-91. https://doi.org/10.1523/JNEUROSCI.1302-06.2006.

Haustein, Martin D, Sebastian Kracun, Xiao-Hong Lu, Tiffany Shih, Olan Jackson-Weaver, Xiaoping Tong, Ji $\mathrm{Xu}$, et al. 2014. "Conditions and Constraints for Astrocyte Calcium Signaling in the Hippocampal Mossy Fiber Pathway." Neuron 82 (2): 413-29. https://doi.org/10.1016/j.neuron.2014.02.041.

Heller, Janosch P., and Dmitri A. Rusakov. 2015. "Morphological Plasticity of Astroglia: Understanding Synaptic Microenvironment." Glia 63 (12): 2133. https://doi.org/10.1002/GLIA.22821.

Hille, Bertil. 2001. Ion Channel of Excitable Membranes. 3rd ed. Sinauer.

Hove-Madsen, L., P. -F. Méry, J. Jurevičius, A. V. Skeberdis, and R. Fischmeister. 1996. "Regulation of Myocardial Calcium Channels by Cyclic AMP Metabolism." Basic Research in Cardiology 91 (S1): 1-8. https://doi.org/10.1007/BF00795355.

Iino, Masae, Kaori Goto, Wataru Kakegawa, Haruo Okado, Makoto Sudo, Shogo Ishiuchi, Akiko Miwa, et al. 2001. "Glia-Synapse Interaction Through Ca2+Permeable AMPA Receptors in Bergmann Glia." Science 292 (5518): 926-29.

https://doi.org/10.1126/science.1058827.

Kamijo, Satoshi, Yuichiro Ishii, Shin-ichiro Horigane, Kanzo Suzuki, Masamichi Ohkura, Junichi Nakai, Hajime Fujii, Sayaka Takemoto-Kimura, and Haruhiko Bito. 2018. "A Critical Neurodevelopmental Role for LType Voltage-Gated Calcium Channels in Neurite Extension and Radial Migration." The Journal of Neuroscience 38 (24): 5551-66.

https://doi.org/10.1523/JNEUROSCI.2357-17.2018.

Kingston, Robert E., Claudia A. Chen, and John K. Rose. 2003. "Calcium Phosphate Transfection." Current Protocols in Molecular Biology 63 (1): 9.1.1-9.1.11. https://doi.org/10.1002/0471142727.mb0901s63.

Kirischuk, S, H Kettenmann, and A Verkhratsky. 1997. "Na+/Ca2+ Exchanger Modulates Kainate-Triggered Ca2+ Signaling in Bergmann Glial Cells in Situ." FASEB Journal: Official Publication of the Federation of American Societies for Experimental Biology 11 (7): 566-72. https://doi.org/10.1096/fasebj.11.7.9212080.

Krzywinski, Martin, and Naomi Altman. 2014. "Analysis of Variance and Blocking." Nature Methods 11 (7): 699700. https://doi.org/10.1038/nmeth.3005.

Kurshan, Peri T, Asli Oztan, and Thomas L Schwarz. 2009. "Presynaptic A2 $\delta$-3 Is Required for Synaptic Morphogenesis Independent of Its Ca2+-Channel Functions." Nature Neuroscience 12 (11): 1415-23. https://doi.org/10.1038/nn.2417.

Latour, Isabelle, Jawed Hamid, Aaron M. Beedle, Gerald W. Zamponi, and Brian A. Macvicar. 2003. "Expression of Voltage-Gated Ca2+ Channel Subtypes in Cultured Astrocytes." Glia 41 (4): 347-53. https://doi.org/10.1002/glia.10162.

Lepski, Guilherme, Cinthia E. Jannes, Guido Nikkhah, and Josef Bischofberger. 2013. "CAMP Promotes the Differentiation of Neural Progenitor Cells in Vitro via Modulation of Voltage-Gated Calcium Channels." Frontiers in Cellular Neuroscience 7 (September): 155. https://doi.org/10.3389/fncel.2013.00155.

Matsui, Ko, Craig E Jahr, and Maria E Rubio. 2005. "High-Concentration Rapid Transients of Glutamate Mediate Neural-Glial Communication via Ectopic Release." Journal of Neuroscience 25 (33): 7538-47. http://www.jneurosci.org/content/jneuro/25/33/7538 .full.pdf.

Matyash, Vitali, and Helmut Kettenmann. 2010. "Heterogeneity in Astrocyte Morphology and Physiology." Brain Research Reviews 63 (1-2): 2-10. https://doi.org/10.1016/j.brainresrev.2009.12.001.

Molotkov, Dmitry, Svetlana Zobova, Jose Miguel Arcas, and Leonard Khiroug. 2013. "Calcium-Induced Outgrowth of Astrocytic Peripheral Processes Requires Actin Binding by Profilin-1." Cell Calcium 53 (5-6): 33848. https://doi.org/10.1016/J.CECA.2013.03.001.

Oberheim, Nancy Ann, Xiaohai Wang, Steven Goldman, and Maiken Nedergaard. 2006. "Astrocytic Complexity Distinguishes the Human Brain." Trends in Neurosciences 29 (10): 547-53. https://doi.org/10.1016/J.TINS.2006.08.004.

Panatier, A., and R. Robitaille. 2016. "Astrocytic MGluR5 and the Tripartite Synapse.” Neuroscience 323 (May): 2934.

https://doi.org/10.1016/J.NEUROSCIENCE.2015.03. 063.

Perez-Alvarez, Alberto, Marta Navarrete, Ana Covelo, Eduardo D Martin, and Alfonso Araque. 2014. "Structural and Functional Plasticity of Astrocyte Processes and Dendritic Spine Interactions." The Journal of Neuroscience : The Official Journal of the Society for Neuroscience 34 (38): 12738-44. https://doi.org/10.1523/JNEUROSCI.2401-14.2014.

Porter, J T, and K D McCarthy. 1996. "Hippocampal Astrocytes in Situ Respond to Glutamate Released from Synaptic Terminals." The Journal of Neuroscience: The Official Journal of the Society for Neuroscience 16 (16): 507381. https://doi.org/10.1523/JNEUROSCI.16-16- 
05073.1996.

Robinson, Philip, Sarah Etheridge, Lele Song, Paul Armenise, Owen T. Jones, and Elizabeth M. Fitzgerald. 2010. "Formation of N-Type (Cav2.2) Voltage-Gated Calcium Channel Membrane Microdomains: Lipid Raft Association and Clustering." Cell Calcium 48 (4): 18394. https://doi.org/10.1016/J.CECA.2010.08.006.

Robinson, Philip, Sarah Etheridge, Lele Song, Riddhi Shah, Elizabeth M. Fitzgerald, and Owen T. Jones. 2011. "Targeting of Voltage-Gated Calcium Channel A2 $\delta$-1 Subunit to Lipid Rafts Is Independent from a GPI-Anchoring Motif." PLoS ONE 6 (6). https://doi.org/10.1371/journal.pone.0019802.

Rojas, Héctor, Claudia Colina, Magaly Ramos, Gustavo Benaim, Erica H. Jaffe, Carlo Caputo, and Reinaldo DiPolo. 2007. "Na+ Entry via Glutamate Transporter Activates the Reverse $\mathrm{Na}+/ \mathrm{Ca} 2+$ Exchange and Triggers -Induced Ca2+ Release in Rat Cerebellar Type1 Astrocytes." Journal of Neurochemistry 100 (5): 11881202. https://doi.org/10.1111/j.14714159.2006.04303.x.

Rose, Christine R., Lisa Felix, Andre Zeug, Dirk Dietrich, Andreas Reiner, and Christian Henneberger. 2018. "Astroglial Glutamate Signaling and Uptake in the Hippocampus." Frontiers in Molecular Neuroscience 10 (January): 1-20. https://doi.org/10.3389/fnmol.2017.00451.

Santello, Mirko, Nicolas Toni, and Andrea Volterra. 2019. "Astrocyte Function from Information Processing to Cognition and Cognitive Impairment." Nature Neuroscience 22 (2): 154-66. https://doi.org/10.1038/s41593-018-0325-8.

Shaw, Robin M., and Henry M. Colecraft. 2013. "L-Type Calcium Channel Targeting and Local Signalling in Cardiac Myocytes." Cardiovascular Research 98 (2): 17786. https://doi.org/10.1093/cvr/cvt021.

Song, Lele, Italo Espinoza-Fuenzalida, Sarah Etheridge, Owen Jones, and Elizabeth Fitzgerald. 2015. "The RDomain: Identification of an N-Terminal Region of the $\alpha_{2} \delta-1$ Subunit Which Is Necessary and Sufficient for Its Effects on $\mathrm{Ca}_{\mathrm{v}} 2.2$ Calcium Currents." Current Molecular Pharmacology 8 (2): 169-79. https://doi.org/10.2174/1874467208666150507104555.

Striessnig, Jörg, and Alexandra Koschak. 2008. "Channels Exploring the Function and Pharmacotherapeutic Potential -of Voltage-Gated Ca 2+ Channels with GeneKnockout Models." https://doi.org/10.4161/chan.2.4.5847.

Sun, Wei, Evan McConnell, Jean-Francois Pare, Qiwu Xu, Michael Chen, Weiguo Peng, Ditte Lovatt, Xiaoning Han, Yoland Smith, and Maiken Nedergaard. 2013. "Glutamate-Dependent Neuroglial Calcium Signaling Differs between Young and Adult Brain." Science (New York, N. Y.) 339 (6116): 197-200.

https://doi.org/10.1126/science. 1226740.

Swanson, Geoffrey T, and Ryuichi Sakai. 2009. "Ligands for Ionotropic Glutamate Receptors." Progress in Molecular and Subcellular Biology 46: 123-57. https://doi.org/10.1007/978-3-540-87895-7_5.

Taylor, Charles P., Timothy Angelotti, and Eric Fauman. 2007. "Pharmacology and Mechanism of Action of Pregabalin: The Calcium Channel A2- $\delta$ (Alpha2-Delta) Subunit as a Target for Antiepileptic Drug Discovery." Epilepsy Research 73 (2): 137-50. https://doi.org/10.1016/J.EPLEPSYRES.2006.09.008.

Thul, Peter J, Lovisa Åkesson, Mikaela Wiking, Diana Mahdessian, Aikaterini Geladaki, Hammou Ait Blal, Tove Alm, et al. 2017. "A Subcellular Map of the Human Proteome." Science (New York, N.Y.) 356 (6340): eaal3321. https://doi.org/10.1126/science.aal3321.

Uhlen, Mathias, Per Oksvold, Linn Fagerberg, Emma Lundberg, Kalle Jonasson, Mattias Forsberg, Martin Zwahlen, et al. 2010. "Towards a Knowledge-Based Human Protein Atlas." Nature Biotechnology 28 (12): 1248-50. https://doi.org/10.1038/nbt1210-1248.

Verkhratsky, Alexei, and Frank Kirchhoff. 2007. "Glutamate-Mediated Neuronal-Glial Transmission." In Journal of Anatomy, 210:651-60. John Wiley \& Sons, Ltd (10.1111). https://doi.org/10.1111/j.14697580.2007.00734.x.

Wang, F., T. Du, C. Liang, A. Verkhratsky, and L. Peng. 2015. "Ammonium Increases $\mathrm{Ca}^{2+}$ Signalling and Upregulates Expression of $\mathrm{Ca}_{\mathrm{v}} 1.2$ Gene in Astrocytes in Primary Cultures and in the in Vivo Brain." Acta Physiologica 214 (2): 261-74. https://doi.org/10.1111/apha.12500.

Westenbroek, Ruth E., Suzanne B. Bausch, Richard C. S. Lin, Joanne E. Franck, Jeffery L. Noebels, and William A. Catterall. 1998. "Upregulation of L-Type Ca2+ Channels in Reactive Astrocytes after Brain Injury, Hypomyelination, and Ischemia." Journal of Neuroscience 18 (7): 2321-34. https://doi.org/10.1523/JNEUROSCI.18-0702321.1998.

Westermark, Bengt. 1973. "The Deficient Densitydependent Growth Control of Human Malignant Glioma Cells and Virus-transformed Glia-like Cells in Culture." International Journal of Cancer 12 (2): 438-51. https://doi.org/10.1002/ijc.2910120215.

Woolley, C S, E Gould, M Frankfurt, and B S McEwen. 1990. "Naturally Occurring Fluctuation in Dendritic Spine Density on Adult Hippocampal Pyramidal Neurons." The Journal of Neuroscience : The Official Journal of the Society for Neuroscience 10 (12): 4035-39. https://doi.org/10436047.

Yoshizumi, Masaru, James C. Eisenach, and Ken-ichiro Hayashida. 2012. "Riluzole and Gabapentinoids Activate Glutamate Transporters to Facilitate Glutamate-Induced Glutamate Release from Cultured Astrocytes." European Journal of Pharmacology 677 (1-3): 87-92. https://doi.org/10.1016/J.EJPHAR.2011.12.015.

Youn, Dong-ho, Gábor Gerber, and William A Sather. 2013. "Ionotropic Glutamate Receptors and VoltageGated $\mathrm{Ca}^{2+}$ Channels in Long-Term Potentiation of Spinal Dorsal Horn Synapses and Pain 
bioRxiv preprint doi: https://doi.org/10.1101/2019.12.15.875799; this version posted December 16, 2019. The copyright holder for this preprint (which was not certified by peer review) is the author/funder, who has granted bioRxiv a license to display the preprint in perpetuity. It is made available under aCC-BY-NC-ND 4.0 International license.

Hypersensitivity." Neural Plasticity 2013: 654257. https://doi.org/10.1155/2013/654257.

Zamponi, Gerald W, Joerg Striessnig, Alexandra Koschak, and Annette C Dolphin. 2015. "The Physiology, Pathology, and Pharmacology of Voltage-Gated Calcium Channels and Their Future Therapeutic Potential." PHARMACOLOGICAL REVIEWS Pharmacol Rev 67: 821-70. https://doi.org/10.1124/pr.114.009654.

Zatkova, M., A. Reichova, Z. Bacova, V. Strbak, A. Kiss, and J. Bakos. 2018. "Neurite Outgrowth Stimulated by Oxytocin Is Modulated by Inhibition of the Calcium Voltage-Gated Channels." Cellular and Molecular Neurobiology 38 (1): 371-78.

https://doi.org/10.1007/s10571-017-0503-3. 